\title{
Crack Detection and Recognition Model of Parts Based on Machine Vision
}

\author{
Dan Li ${ }^{1, *}$, Daihong Jiang ${ }^{1}$, Rong Bao ${ }^{1}$, Lei Chen ${ }^{1}$ and Matthew Keith Kerns ${ }^{2}$ \\ ${ }^{1}$ Key Laboratory of Intelligent Industrial Control Technology of Jiangsu Province, Xuzhou University of Technology, Xuzhou \\ Jiangsu, 221000, China \\ ${ }^{2}$ G2K Equipment and Services LLC,2209 Spruce Cir, Mckinney, TX, 75071, United States
}

Received 7 July 2019; Accepted 11 October 2019

\begin{abstract}
The traditional manual crack detection method for parts is inefficient, subjective, and has low accuracy. Laser and radar equipment is accurate but costly. Thus, image processing methods based on machine vision are widely used owing to the rapid detection speed and low cost. However, these methods are inappropriate to a low-contrast, high-noise, and poorresolution environment. To obtain accurate detection results in complex environments, this study proposed a new automatic crack identification model. Image quality was enhanced using the multi-scale Retinex algorithm modified by a three-sided filter. The algorithm for spectral clustering was employed to segment the cracks, thereby potentially preserving the relevant information between the samples. Pixel interference regions were removed on the bases of shape feature and contour information. Target feature vectors were extracted according to the characteristics of the crack, and five features of the crack image were constructed. The SVDD(support vector data description) classifier was constructed by using machine learning methods to automatically distinguish between crack and non-crack structures. The crack direction was identified on the basis of the projections in the vertical and horizontal directions. The accuracy of the model was verified by experiments. Results indicate that the crack images with a size of $300 \times 400$ have good segmentation effect and low complexity through the clustering algorithm, while the cluster number is equal to 2 . Noise interference can be further reduced by setting the crack area between $2 \%$ to $28 \%$. Moreover, the detected crack width can be accurate to 1 pixel. The crack and non-crack areas can be classified using the SVDD classifier without large samples. The proposed method provides good reference for inspection platform construction and state assessment.
\end{abstract}

Keywords: Crack detection, Multi-scale Retinex, Spectral clustering; SVDD classifier

\section{Introduction}

Crack is an important parameter that directly affects the quality and safety of finished products. The early detection and maintenance of cracks can effectively avoid further damage $[1,2]$. The method of manually detecting cracks is subjective, inefficient, and susceptible to inspector fatigue. Although cracks can be detected accurately using lasers and radars, such methods will cost substantial money and high power. The development of machine vision and sensor technologies has resulted in the prevalence of automatic crack detection based on digital image processing technology. Numerous studies have been conducted on machine visionbased crack detection methods [3-5]. However, the rapid speed of current crack detection methods is immaterial because such techniques are unsuitable for a complicated situation, in which the image contrast is low, the multi-texture shape is irregular, and the noise is complicated. Therefore, detecting and identifying cracks accurately in an interference environment are urgent problems that should be solved.

This study focuses on the accuracy of algorithms in complex scenarios and proposes a new crack detection and classification model that features image enhancement and crack interference removal, extraction, and classification. The

*E-mail address: lidanonline@163.com

ISSN: $1791-2377$ @ 2019 School of Science, IHU. All rights reserved.

doi:10.25103/iestr.125.17 proposed model can accurately identify cracks, obtain parameter information, and provide reference for further damage.

\section{State of the art}

At present, numerous studies have been conducted on crack detection, identification and, extraction. Li [6] proposed a crack segmentation algorithm based on threshold segmentation for adjacent differential histograms to accurately extract crack information from images. Although this algorithm is simple and easy to use, it does not consider the influence of noise, texture, and illumination on crack recognition, thereby leading to difficulty in achieving stable results. Schmugge [7] used depth learning method to divide image pixels into crack and background and used crack detection as a segmentation task. However, the result yielded poor accuracy. Achanta [8,9] achieved good results for the salient region detection of images in the Berkeley database but failed to work well for the continuity of the detected cracks. Oliverira [10] proposed crack pixel recognition and classification methods through unsupervised learning system. Although this method has good detection and classification results, difficulties were experience when handling fine cracks. Nguyen [11] divided a crack image into sub-images, performed feature extraction thereafter to construct feature vectors, and trained to identify sub-images and detect cracks. 
However, manually designing and constructing feature vectors are complex, thereby needing different methods for feature extraction according to different backgrounds. Therefore, the aforementioned method remains unsuitable for crack detection and identification. Zhang [12] used deep learning method to perform block-based classification crack detection. However, the proposed method leads to inconvenience in detecting and sensitive to block size. Valença [13] used triangles to divide an image, high-pass filters to enhance an image, and binarized the image through the Otsu method. Thereafter, the aforementioned research used the morphological algorithm to synthesize the rectangular image, thereby detecting the cracks by eliminating the discontinuity of the surface. However, the identification of small cracks is not evident. Koch [14] proposed a method based on computer vision technology for crack detection and evaluation. The proposed method has high detection accuracy and avoids cracks in the detected cracks but is unsuitable for complex environments, such as those with illumination. $\mathrm{Hu}$ [15] used crack texture based on texture analysis to detect cracks and backgrounds. To separate the cracks and backgrounds, local binary pattern operators were employed to determine whether each pixel belongs to a crack. However, this method does not consider local neighborhood information, thereby resulting in failure to accurately detect cracks with uneven intensity. McCormack [16] used the domain weighted averaging method to preprocess a gray image, and combined the local threshold method and shape filtering thereafter to obtain the crack detection algorithm. Deep convolution generative confrontation network was used to increase the crack data set, thereby effectively alleviating the under-fitting phenomenon and improving the FC-Dense Net103 model [17]. The model parameters are few and its accuracy should be further improved. Prasanna [18] scanned images of buildings by remotely manipulating the robot Seekur and used the STRUM classifier to detect cracks. However, the robustness of a complex environment with dark and uneven illumination is not high. Zhao [19] made corresponding improvements to VGGNet, which connected the original networks conv1_1, conv2_2, conv3_3, conv4_3 directly to the last convolution layer. However, the improved version experienced difficulty in accurately identifying the considerably complex targets in the image. Cha [20] used a fully automated convolutional neural network (CNN) to extract and classify pavement cracks, and compared the effects of the traditional Canny and Sobel operators on edge extraction. Nevertheless, directly applying the existing deep learning algorithm to crack identification will yield such problems as long training time and low prediction accuracy. Zou [21] proposed a crack detection algorithm based on minimum path selection for low crack contrast and poor continuity, thereby providing improved solution to the problem of crack detection and extraction under low SNR conditions. However, this algorithm does not consider the extraction of cracks under complex conditions, such as those with low illumination and uneven crack distribution.

The preceding discussion indicated improved detection and recognition effects in an environment with high resolution, low noise interference, and uniform illumination. However, the proposed models are highly susceptible to lowcontrast, multi-texture, uneven-crack situations. In the current study, the Retinex method can be improved by using a threeedge filter, which is employed to enhance image quality. Moreover, the image is segmented using a spectral clustering algorithm to complete the initial crack detection. The shape feature and contour information are used to remove the interference information, thereby ensuring the long and linear features of cracks. To identify the crack and non-crack areas, the five characteristics of an SVDD(support vector data description) classifier are selected to make effective evaluation. In addition, the transverse longitudinal crack can be assessed using the crack projection, which provides basis for automatic detection, identification, and early warning.

The remainder of this study is organized as follows. Section 3 establishes the crack detection and identification model, which proposes the methods for crack segmentation, image classification, and discrimination of transverse and longitudinal cracks. Section 4 discusses the applicability of the proposed method through case studies. Section 5 provides the conclusions.

\section{Methodology}

\subsection{Segmentation of crack images}

\subsubsection{Image preprocessing}

This study aims to solve the problem related to the collected local crack image having low contrast, high noise, blurred detail, dark brightness and uneven illumination environment, which will affect the quality of images. Thus, a three-edge filter is used to improve the multi-scale Retinex method for enhancing image quality [22], thereby improving the optimization effect for later experiments.

On the basis of a central surround idea, a Gaussian filter is utilized to estimate the incident component, which has good consistency of color fidelity, dynamic compression range, and local detail. Tri-edge filter maintains a stepped image edge feature that can enhance the details of the highlight and dark areas. The extraction of the illuminance component maintains a high contrast edge, thereby avoiding the appearance of passivation or blurred edges when images are enhanced. The proposed method solves the problem that the traditional direct contrast enhancement method is sensitive to noise. Moreover, the homomorphic filtering enhancement method results in inconspicuous contrast.

\subsubsection{Crack segmentation based on spectral clustering algorithm}

The traditional K-means clustering algorithm [23] is a typical algorithm that uses distance as the evaluation index to classify data. This algorithm can adapt and find the best clustering center through iterative calculation. The clustering operation is performed by calculating the metric distance for all data. However, the distance between the samples is no longer evident when facing high-dimensional data, and the complexities of time and space of the algorithm are high.

Spectral clustering algorithm $[24,25]$ is one of the subspace clustering methods. This algorithm is based on the idea of graph theory that considers the sample as a point in space and connects the vertices thereafter by edges. The weight of each edge describes the similarity of the points at the ends of the edge (i.e., similarity among the corresponding samples). If two samples have high similarity, then the corresponding weights are large; otherwise, the corresponding weights are smaller. Thereafter, the graph constructed using the aforementioned method is divided into a series of subgraphs. The development of these subgraphs satisfy the following objectives: the sum of the edge weights in the subgraph is the largest and the sum of the edge weights between the subgraphs is the smallest. Lastly, each subgraph corresponds to a cluster. The algorithm for spectral clustering 
Dan Li, Daihong Jiang, Rong Bao, Lei Chen and Matthew Keith Kerns/

Journal of Engineering Science and Technology Review 12 (5) (2019) 148 - 156

reduces the dimension by mapping the relationship between the high-dimensional data on a two-dimensional map. After comparing with distance-based algorithms, such as K-means, spectral clustering can considerably preserve the relevant information between samples and is substantially targeted and efficient.

The full connection based on the Gaussian kernel distance. The Ncut chart introduces the algorithm flow of spectral clustering. The pseudocode of the turbulence operation works as follows:

Input: Sample set $\mathrm{X}=\left[\mathrm{x}_{1}, \mathrm{x}_{2}, \mathrm{x}_{3}, \ldots, \mathrm{x}_{\mathrm{n}}\right]$; Number of clusters: $K$;

Output: Cluster division $C=\left(c_{1}, c_{2}, c_{3}, \ldots, c_{k}\right)$;

Step1) Calculate Affinity matrix $W$;

Step2) Calculate Degree matrix $D$;

Step3) Calculate Laplace matrix $L$;

Step4) Construct a standardized Laplace matrix $D^{-\frac{1}{2}} L D^{\frac{1}{2}}$;

Step5) Calculate the first $K$ eigenvalues and the corresponding eigenvectors of $D^{-\frac{1}{2}} L D^{\frac{1}{2}}$;

Step6) Construct the feature matrix $F$ from the feature vector and normalize it by row;

Step7) Clustering $n$ samples in $F$ using K-means algorithm; Step8) Get cluster division $C$.

For a given data set $X=\left[x_{1}, x_{2}, x_{3}, \ldots, x_{n}\right]$, mapping $X$ to a point set $V$. The matrix $W$ is used to represent the edge weight between points and $W$ is called the adjacency matrix. In spectral clustering, three methods are typically used to measure the similarity between samples: $\varepsilon$-nearest neighbor, $K$-nearest neighbor, and full join methods. This study used the full join method.

The full join method connects each sample to other samples, although the mere weights of the connections are different between the points. A complete graph in point set $V$ is constructed from the perspective of graph theory. Kernel functions are generally used to determine the weights between edges. The commonly used kernel functions are the polynomial, Gaussian, and Sigmoid kernel functions. When using a Gaussian kernel function, the adjacency matrix can be expressed as follows:

$w_{i j}=\exp \left(-\frac{\left\|x_{i}-x_{j}\right\|_{2}^{2}}{2 \sigma^{2}}\right)$

The Gaussian kernel function is the most common and widespread in the practical application of the full connection method. For any point vi in a graph, the sum of the weights of all edges connected to such a point is defined as di:

$$
d_{i}=\sum_{j=1}^{n} w_{i j}
$$

An $n \times n$ degree matrix $D$ is obtained when $d i$ for each point is calculated:

$$
D=\left[\begin{array}{ccc}
d 1 & \cdots & \cdots \\
\cdots & d 2 & \cdots \\
\vdots & \vdots & \ddots \\
\cdots & \cdots & d n
\end{array}\right]
$$

After obtaining the adjacency matrix $W$ and degree matrix $D$, the Laplace matrix $L$ is calculated from the Laplacian matrix definition $L=D-W$. For any vector $\boldsymbol{f}$, the following relationship is satisfied:

$$
f^{T} L f=\frac{1}{2} \sum_{i, j=1}^{n} w_{i j}\left(f_{i}-f_{j}\right)^{2}
$$

When performing an undirected cut, the minimum cut problem is often caused by various factors, thereby making the cut effect unsatisfactory. In this regard, certain restrictions on the size of each subgraph are needed when cutting the graph. In general, the two methods of cutting are the RatioCut and Ncut. This study used the Ncut charts.

For the cut graph of the undirected graph $G(V, E)$, the cut target is to cut all the points in the graph into $K$ unconnected subgraph. $A_{i}(1 \leq i \leq K)$ is utilized to represent the point set of subgraph $i$, which has the following two properties:
a) $A_{i} \cap A_{j}=\varnothing$
b) $A_{1} \cup A_{2} \cup \ldots \cup A_{k}=V$

For point sets $A$ and $B$ of any two subgraphs, the weights of the cut between $A$ and $B$ are defined as follows:

$$
W(A, B)=\sum_{i \in A, j \in B} w_{i j}
$$

Given the preceding theoretical basis, the Ncut chart can be defined as follows:

$\operatorname{Ncut}\left(A_{1}, A_{2}, \ldots, A_{K}\right)=\frac{1}{2} \sum \frac{W\left(A_{i}, \bar{A}_{i}\right)}{\operatorname{vol}\left(A_{i}\right)}$

where $\bar{A}_{i}$ is a complement of $A_{i}$. Apart from $A_{i}$, the union of all subsets in $v$ is represented as $\operatorname{vol}(A):=\sum_{i \in A} d_{i}$. Thereafter, the Ncut function is minimized and the indicator vector $h_{j} \in\left\{h_{1}, h_{2}, \ldots, h_{k}\right\}, 1 \leq j \leq k$ is introduced. For any vector $h_{j}$, an $n$-dimensional vector ( $n$ is the number of samples) is evident, and $h_{i j}$ is defined as follows:

$h_{i j}=\left\{\begin{array}{c}0, v_{i} \notin A_{j} \\ \frac{1}{\sqrt{\operatorname{vol}\left(A_{j}\right)}}, v_{i} \in A_{j}\end{array}\right.$

Formula (7) indicates that for $h_{i}^{T} L h_{i}$, the following formula is derived: 


$$
\begin{aligned}
& h_{i}^{T} L h_{i}=\frac{1}{2} \sum_{m=1} \sum_{n=1} w_{m n}\left(h_{i m}-h_{i n}\right)^{2} \\
& =\frac{1}{2}\left(\sum_{m \in A_{i}, n \notin A_{i}} w_{m n}\left(\frac{1}{\sqrt{\operatorname{vol}\left(A_{i}\right)}}-0\right)^{2}+\sum_{m \notin A_{i}, n \in A_{i}} w_{m n}\left(0-\frac{1}{\sqrt{\operatorname{vol}\left(A_{i}\right)}}\right)^{2}\right) \\
& =\frac{1}{2}\left(\sum_{m \in A_{i}, n \notin A_{i}} w_{m n} \frac{1}{\operatorname{vol}\left(A_{i}\right)}+\sum_{m \notin A_{i}, n \in A_{i}} w_{m n} \frac{1}{\operatorname{vol}\left(A_{i}\right)}\right) \\
& =\frac{1}{2}\left(\operatorname{cut}\left(A_{i}, \bar{A}_{i}\right) \frac{1}{\operatorname{vol}\left(A_{i}\right)}+\operatorname{cut}\left(\bar{A}_{i}, A_{i}\right) \frac{1}{\operatorname{vol}\left(A_{i}\right)}\right) \\
& =\frac{\operatorname{cut}\left(A_{i}, \bar{A}_{i}\right)}{\operatorname{vol}\left(A_{i}\right)}
\end{aligned}
$$

The optimization objective function is as follows:

$$
\operatorname{Ncut}\left(A_{1}, A_{2}, \ldots, A_{k}\right)=\sum_{i=1}^{k} h_{i}^{T} L h_{i}=\sum_{i=1}^{k}\left(H^{T} L H\right)_{i i}=\operatorname{tr}\left(H^{T} L H\right)
$$

Let $H=D^{-\frac{1}{2}} F \quad$.Thereafter: $\quad H^{T} L H=F^{T} D^{-\frac{1}{2}} L D^{\frac{1}{2}} F$, $H^{T} D H=F^{T} F=I$. The optimization goal is as follows:

$\underbrace{\arg \min }_{F} \operatorname{tr}\left(F^{T} D^{-\frac{1}{2}} L D^{\frac{1}{2}} F\right)$ s.t. $F^{T} F=I$

Lastly, the first $K$ eigenvalues and the corresponding eigenvectors in $D^{-\frac{1}{2}} L D^{\frac{1}{2}}$ are determined. Given that the maximum eigenvalue of the matrix is faster and more efficient than the minimum eigenvalue, the problem of finding the first $K$ minimum eigenvalues and corresponding eigenvectors of $D^{-\frac{1}{2}} L D^{\frac{1}{2}}$ has been transformed into the first $K$ largest eigenvalues and the corresponding eigenvectors of the $D^{-\frac{1}{2}} W D^{\frac{1}{2}}$. According to the relevant characteristics of the singular value, $D^{-\frac{1}{2}} W D^{\frac{1}{2}}$ should be decomposed to obtain the result. After normalizing the results, the feature matrix $F$ is obtained and the final clustering task is completed for $F$ by using the traditional clustering algorithm.

Through the spectral clustering algorithm, image pixels can be divided into two types of classification samples: crack and background pixel sets. Thus, the binary image of the preliminary crack segmentation is obtained.

\subsection{Identification and removal of interference information based on morphology and contour}

After the image is processed using the spectral clustering algorithm, the obtained binary image contains extensive interference information, such as small noise and nonlinear strip shape interference. Therefore, a corresponding filtering method according to the characteristics of "pseudo-cracks" should be designed to improve the recognition rate of cracks.

\subsubsection{Disturbance removal of connected area based on morphology}

In the basic operation of morphology, the expansion fills the edges or the internal holes, thereby enlarging the entire crack profile. Corrosion can remove the burrs and small protrusions on the crack target or can corrode the fine joint between the cracks. Hence, the crack breaks initially and erodes thereafter. The closed operation can smoothen the contour of the image and fill the crack. Moreover, the change in the crack boundary is guaranteed to be small.
After dividing all the connected domains in the image, the size of pixels included in the connected domain can be obtained. Only a few pixels are observed in the small noise areas, and a threshold $T$ can be set to discard the area where the connected domain is below the threshold. In this study, the experiments conducted involving different images indicated that the threshold value is better when $T=20$. That is, all connected domains below 20 pixels in the segmented image are considered noise and should be removed.

3.2.2 Interference removal based on contour information A crack is characterized for its slenderness. Therefore, the contour shape feature of the fracture connected domain is described on the basis of this remarkable feature, and the connected domain set $R_{D}=\left\{r_{1}, r_{2}, \ldots r_{k}\right\}$ is extracted. This step extracts the linear connected domain mainly according to the following two criteria:

1) Find the inscribed circle radius $R_{\text {in }}$ of each connected domain $r_{n}$ in the connected domain set $R_{D}=\left\{r_{1}, r_{2}, \ldots r_{k}\right\} ; R_{i n}$ is below the set threshold $t h_{R}$, which is used to ensure the narrow crack;

2) Find the radius $R_{\text {out }}$ of the circumcircle of each connected domain $r_{n}$ in the connected domain, set $R_{D}=\left\{r_{1}, r_{2}, \ldots r_{k}\right\}$. Calculate the ratio of the inscribed circle radius to the circumscribed circle radius $c=R_{\text {in }} / R_{\text {out }} . c$ should be below the set threshold to ensure that the cracks shapes are long and linear.

\subsection{Feature selection and SVDD classifier construction}

The classification and recognition algorithms in machine learning mainly include decision trees, artificial neural networks, and support vector machines (SVM) [26,27]. In actual use, the majority of the diagnostic models continue to face difficulties in obtaining defective training data and data imbalance. The support vector data description based on SVDD $[28,29]$ inherits the advantages of minimal parameters, rapid learning speed, strong promotion ability, and global optimization and also has the potential to solve online diagnosis problems. When establishing a crack diagnosis model, traditional classification methods often require numerous training samples for adequate training, and image collection requires a lot of time and effort. SVDD overcomes the need for large samples, thereby eliminating the need for defective samples, and can be classified only by normal sample modeling.

\subsubsection{Image feature selection and normalization}

Five features are selected to construct the feature vectors.

(1) Width-to-length ratio of the connected domain. If the ratio $w_{i} / l_{i}$ is large, then it indicates that the width tends to the length, and does not conform to the characteristics of crack slenderness.

(2) Area ratio. The proportion of the connected domain is the ratio of the number of black pixels to the total pixels in the image, which is defined as $F=A / r o w \cdot c o l$,

where $A$ is the connected domain area, rows and col are the number of image rows and columns, respectively, and their product is the total image pixels. The crack pixels generally accounts for $2 \%$ to $28 \%$ of the entire image. Furthermore, the connected domain that does not belong to this interval can be considered a noise sub-block. 
Dan Li, Daihong Jiang, Rong Bao, Lei Chen and Matthew Keith Kerns/

Journal of Engineering Science and Technology Review 12 (5) (2019) 148 - 156

(3) Ratio of the inscribed circle radius to the circumscribed circle radius. $c=R_{\text {in }} / R_{\text {out }}, c$ is below the set threshold $t h$ to ensure that the crack is long and linear.

(4) Eccentricity of the connected domains. It can be used to describe the compactness of the area. The connected domain with an eccentricity of 0 is actually a perfect circle, and 1 is a line segment. The inertia principal axis ratio is calculated and the central moment is used to calculate the eccentricity of any connected domain. The calculation formula is as follows:

$P=\frac{\left(\mu_{28}-\mu_{02}\right)^{2}+4 \mu_{15}}{A}$

where $A$ is the area of the connected area; and $\mu_{28}, \mu_{02}$, and $\mu_{15}$ can be obtained using the central moment formula as follows:

$\mu_{j i}=\sum_{x, y}\left(\operatorname{array}(x, y) \cdot(x-\bar{x})^{j} \cdot(y-\bar{y})^{i}\right)$

In formula (12), where $\bar{x}=\frac{m_{14}}{m_{00}}, \bar{y}=\frac{m_{01}}{m_{00}}$ is the center of gravity of the connected area.

(5) Filling degree of the connected domain. It is often defined as the ratio of the region area $A$ to its outer rectangle area $A r$. In general, the filling degree of cracks is smaller than that of non-cracks mainly because cracks typically have slender structures and the noises have irregular shape. When the direction of a single fracture is nearly vertical or horizontal, cracks and non-cracks are impossible to accurately distinguish from each other. Therefore, the outer circle area of the outer rectangle of the connected region is used instead of the outer rectangle area. Thus, the regional compactness of the connected region can be accurately measured.

Different eigenvector components represent different physical meanings, and the normalization of the eigenvector elements can eliminate this deviation. Let the training sample set be $X=\left[x_{1}, x_{2}, \ldots x_{N}\right]$, and $N$ is the number of samples in the training set. If the maximum, minimum, and average values of each component of the sample are $x_{\text {max }}, x_{\text {min }}$, and $\bar{x}_{\mathrm{i}}$, respectively, it can be normalized according to the formulas of (13) and (14):

$x_{i}=\frac{2 x_{i}-x_{\max }-x_{\min }}{x_{\max }-x_{\min }}$

$x_{i}=\frac{N\left(x_{i}-\bar{x}_{i}\right)}{\sum_{i}^{N}\left(x_{i}-\bar{x}_{i}\right)}$

After normalization, the component values of the training samples are distributed in the interval $[-1,1]$. The data can be normalized in the interval $[0,1]$ using the following formula:

$$
x_{i}=\frac{\left|x_{i}-x_{\min }\right|}{\left|x_{\max }-x_{\min }\right|}
$$

\subsubsection{Construction of the SVDD classifier}

By setting up training sample $X=\left[x_{1}, x_{2}, \ldots x_{N}\right]$, the goal of the support vector description is to find a hypersphere with a center of sphere $a$ and radius of $R$, which contain all or the majority of the target samples $x_{i}$. Meanwhile, the radius of the hypersphere $R$ should be the smallest:

$\left\|x_{i}-a\right\|^{2} \leq R^{2}, i=1,2, \ldots, N$

When relaxation factor $\xi_{i} \geq 0, i=1,2, \ldots, N$ is introduced to enhance the robustness of the classification, the formula (16) is transformed as follows:

$\min f(R, a, x)=R^{2}+C \sum_{i=1}^{N} \xi_{i}, i=1,2, \ldots, N$

$\left\|x_{i}-a\right\|^{2} \leq R^{2}+\xi_{i}, \xi_{i} \geq 0$

Formula (17) shows that a specified constant $C$ is used to control the degree of punishment of the misclassified sample. Under this constraint, the minimum radius of the hypersphere is obtained. That is, the quadratic optimization problem can be converted to Lagrangian extreme value problem as follows:

$$
\begin{aligned}
& L(R, a, \xi, \alpha, \gamma)= \\
& R^{2}+C \sum_{i=1}^{N} \xi_{i}-\sum_{i=1}^{N} \alpha_{i}\left[R^{2}+\xi_{i}-\left(x^{2}-2 a x_{i}+a^{2}\right)\right]-\sum_{i=1}^{N} \gamma_{i} \xi_{i}
\end{aligned}
$$

where $\alpha_{i} \geq 0, \gamma_{i} \geq 0$ is the Lagrangian coefficient and each $x_{i}$ has $\alpha_{i}$, with $\gamma_{i}$ corresponding to it. The partial derivatives of $\mathrm{a}, \mathrm{b}$, and $\mathrm{c}$ obtained, thereby making them all equal to 0 . Thus, $\sum_{i} \alpha_{i}=1$.

$$
\begin{aligned}
& a=\sum_{i} \alpha_{i} x_{i} \\
& C-\alpha_{i}-\gamma_{i}=0
\end{aligned}
$$

After transformation, formula (19) can be written as follows:

$$
L(R, a, \xi, \alpha, \gamma)=\sum_{i=1}^{N} \alpha_{i}\left(x_{i} \cdot x_{j}\right)-\sum_{i=1, j=1}^{N} \alpha_{i} \alpha_{j}\left(x_{i} \cdot x_{j}\right)
$$

The constraint is $\sum_{i} \alpha_{i}=1,0 \leq \alpha_{i} \leq C$.

The optimal solution $\alpha_{i}^{*}$ is derived for this quadratic optimization problem. In the actual calculation, most of the $\alpha_{i}$ will be 0 and a small part will be above 0 . These samples correspond to $\alpha_{i}$, which are above 0 and will become support vectors. The hypersphere radius $R$ can be determined from any of the support vector $x_{k}$ :

$R^{2}=\left(x_{k} \cdot x_{k}\right)-2 \sum_{i=1}^{N} \alpha_{i}\left(x_{i} \cdot x_{k}\right)+\sum_{i=1, j=1}^{N} \alpha_{i} \alpha_{j}\left(x_{i} \cdot x_{j}\right)$ 
Dan Li, Daihong Jiang, Rong Bao, Lei Chen and Matthew Keith Kerns/

Journal of Engineering Science and Technology Review 12 (5) (2019) 148 - 156

or the new sample $z$, if you want to judge whether it belongs to the target sample, then it only needs to calculate the relationship between the distance to the center of the supersphere and $R$. Therefore, the judgment function is as follows:

$$
\|z-a\|^{2} \leq(z \cdot z)-2 \sum_{i=1}^{N} \alpha_{i}\left(x_{i} \cdot z\right)+\sum_{i=1, j=1}^{N} \alpha_{i} \alpha_{j}\left(x_{i} \cdot x_{j}\right) \leq R^{2}
$$

If the judgment function is established, then the new sample $Z$ is accepted as the target sample; otherwise, it is rejected. The Gaussian kernel function is selected, and the expression of the Gaussian kernel is as follows:

$K\left(x, x_{i}\right)=\exp \left\{-\frac{\left\|x-x_{i}\right\|^{2}}{\sigma^{2}}\right\}$

When the Gaussian core radius is $\sigma \rightarrow 0$, there is:

$K\left(x, x_{i}\right) \rightarrow\left\{\begin{array}{c}1, x_{i}=x_{j} \\ 0, x_{i} \neq x_{j}\end{array}\right.$.

When the Gaussian core radius is $\sigma \rightarrow \infty$, there is:

$K\left(x, x_{i}\right) \rightarrow 1, \forall x_{i}, x_{j}$.

When $\sigma \rightarrow 0, R\left(x_{i}\right) \rightarrow 1$, the interval between hypersphere center and and all the sample points in the feature space are the same. The sample points are all located on the sphere. When $\sigma \rightarrow \infty, R\left(x_{i}\right) \rightarrow 0$, all the sample points in the feature space are mapped to one point. Finally, the possible optimal parameters are found by the grid search method, and the accuracy is verified using a cross-validation method.

\subsubsection{Crack direction judgment}
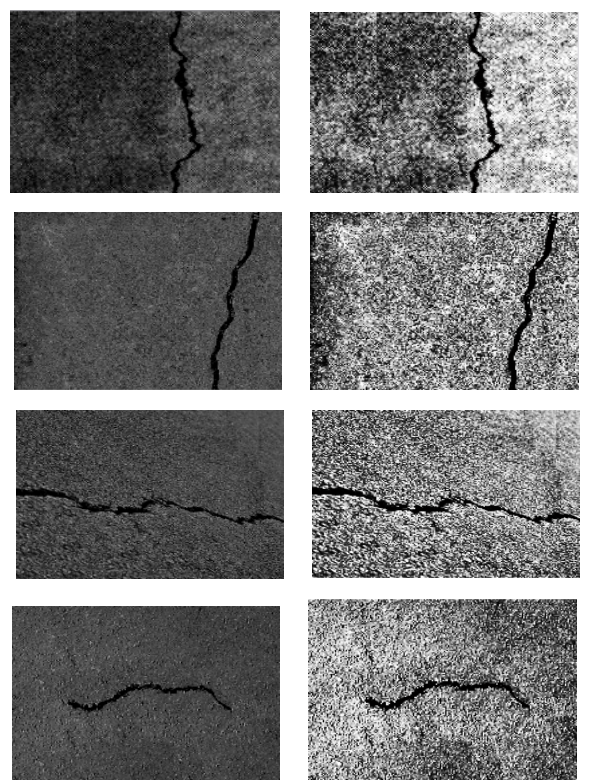

(a)

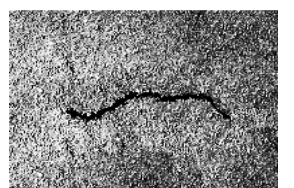

(b)
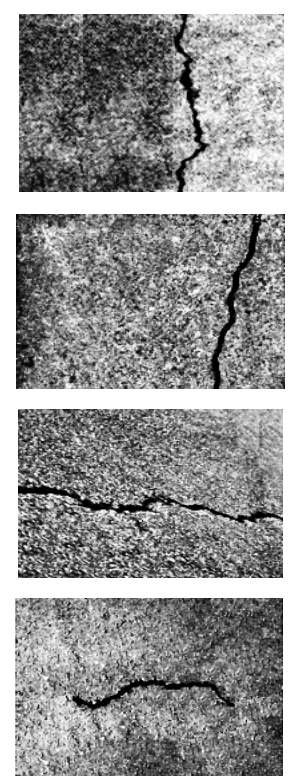

(c)
The cracks appear elongated in geometrical distribution. In this study, the crack direction is judged by the horizontal projection and vertical projection characteristics. In the transverse crack, the length of the non-zero interval of the horizontal projection is longer than the vertical projection. Conversely, the length of the non-zero interval of the vertical projection in the longitudinal crack is longer than the horizontal projection. Meanwhile, the length of the non-zero intervals in two projection directions is calculated. If the length of the horizontal projection interval is greater than the vertical projection, then the crack is a longitudinal crack; otherwise, it is a transverse crack.

\section{Result analysis and discussion}

To verify the effectiveness of the proposed algorithm, the interference information removal, crack extraction location, crack and non-crack classification, and crack type judgment were analyzed. The experimental environment includes Windows 10 operating system, 8G memory, 3.6 GHZ CPU, and MATLAB 2014. The size of images is $300 \times 400$ pixels.

Fig.1 shows that the images are enhanced using the Retinex calculation method based on Section 3.1. Fig.1 (a) shows the original image, in which the color is dark owing to environmental interference, and crack recognition is low. To optimize the image quality (see Fig.1(b)), a Retinex filter is utilized to enhance the preprocess crack image. Compared with the effects of direct contrast enhancement and homomorphic filtering enhancement, Retinex filtering has the best enhancement effect, which improves the contrast of the image and highlights the details. Fig.1 (e) shows the processing result of the subspace spectral clustering algorithm. The image pixels of the component are taken as two types of classification samples: the set of crack pixels of the component image is class 1 and the background pixel set of the component image is class 2 . The value of the number $K$ of the clustering clusters is equal to 2 , and the best clustering result of the crack images are obtained using the spectral clustering algorithm. If the crack and background pixel values are set to 1 and 0 , respectively, then the preliminary crack binary image can be obtained.

Fig. 1. Crack image preprocessing and spectral clustering

Homomorphic filtering.(e) Spectral clustering segmentation
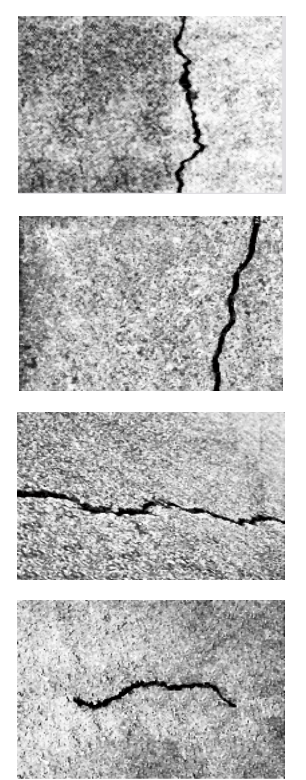

(d)
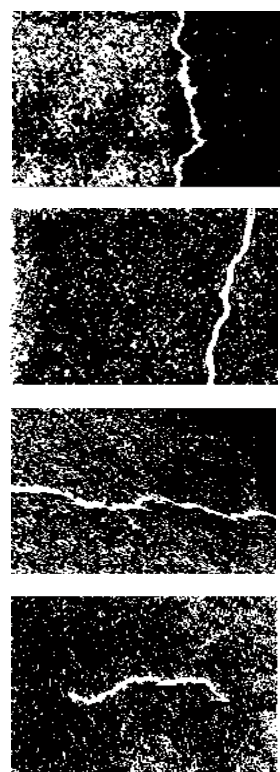

(e) 
Interference can be removed using morphology and contour information, which was introduced in Section 3.2. In the connected area, only a few pixels are included in the small noise areas. The areas that are smaller than the threshold $T$ have been discarded. By experimenting with different images, the threshold $T$ is better when taken at 20 .
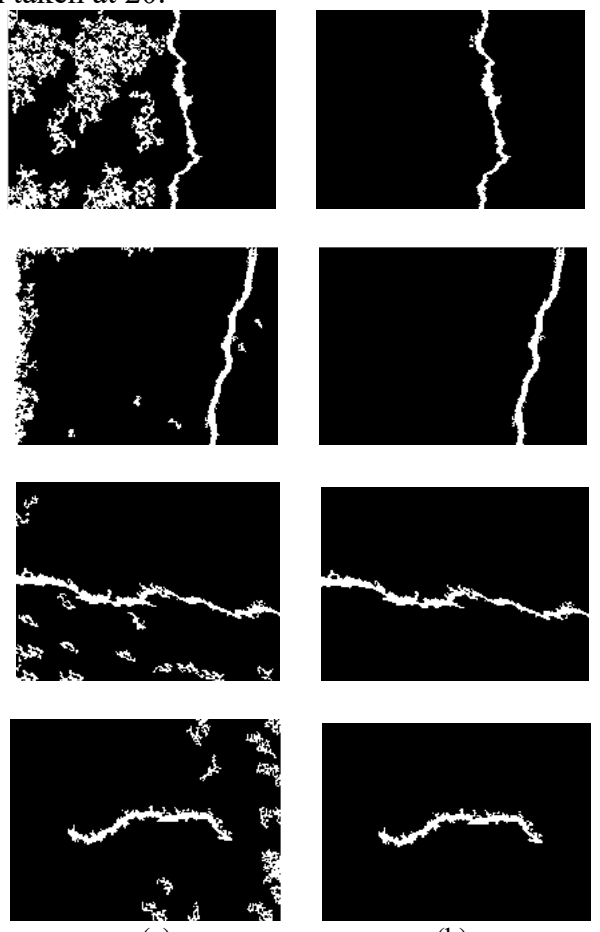

(a)
The linear connected domain is extracted according to the slender characteristics of the crack, and the ratio of the radius of the inscribed circle to the radius of the circumscribed circle is calculated to ensure the crack long and linear.

Fig. 2. Filtering and crack positioning. (a) Area threshold filtering. (b) Shape extreme value filtering. (c) Crack positioning

Fig. 2 (a) and (b) are the results of the area threshold filtering and shape extreme value filtering shown in Figure 1 (e). Small areas or high pixel aggregation density areas were removed. Fig.2 (c) shows the results of the automatic crack location.

To achieve classification of cracks and non-cracks, the SVDD classifier is constructed based on Section 3.3. Five features are used to establish a crack diagnosis model, which includes the width-to-length ratio, area ratio, ratio of the inscribed circle radius to the circumscribed circle radius, eccentricity of the connected domain, and filling degree of the connected domain. This model solves the problem that the defective training data is difficult to obtain in other models. The algorithm for SVDD overcomes the demand for large samples. Cracks and non-cracks can be classified only by normal sample modeling and the direction of the crack images can be judged according to the horizontal and vertical projection characteristics.
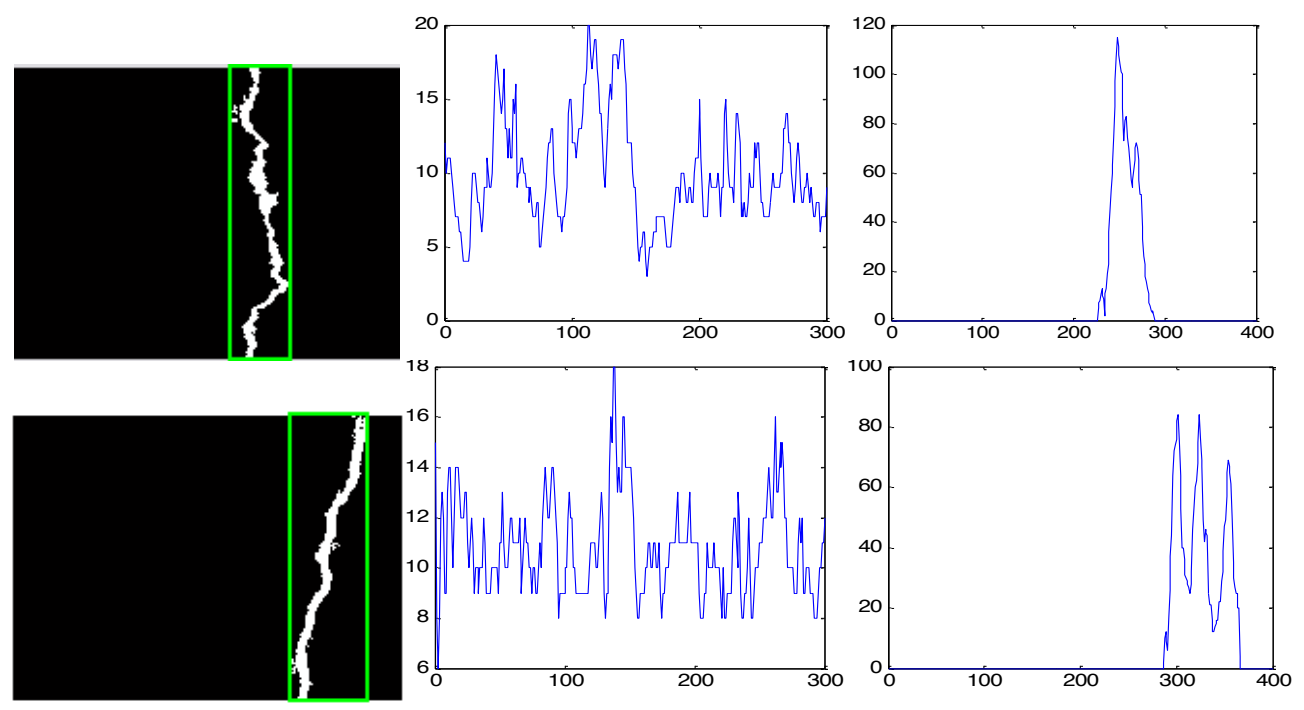
Dan Li, Daihong Jiang, Rong Bao, Lei Chen and Matthew Keith Kerns/

Journal of Engineering Science and Technology Review 12 (5) (2019) 148 - 156
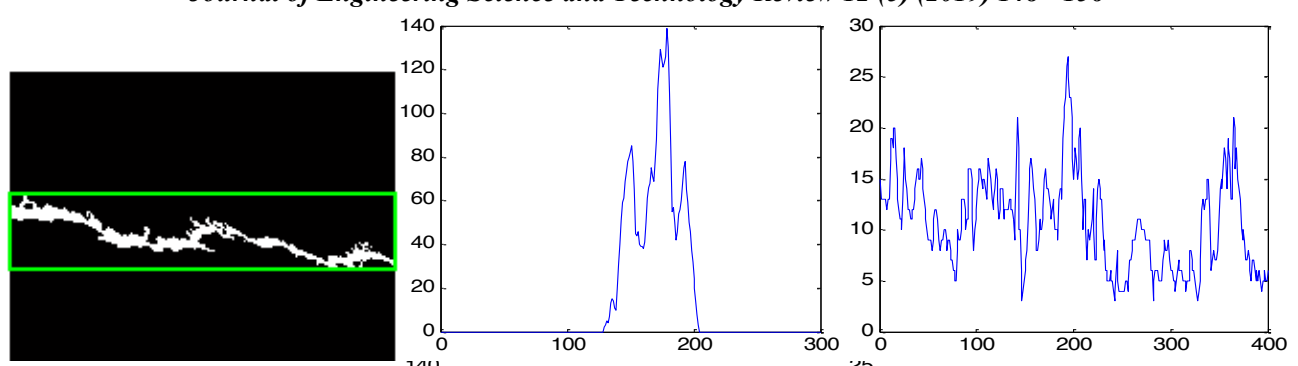

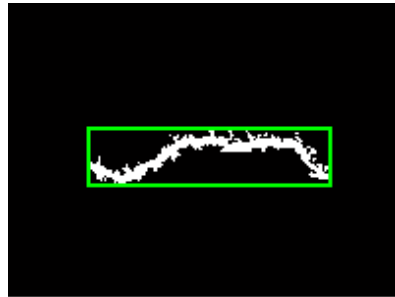

(a)

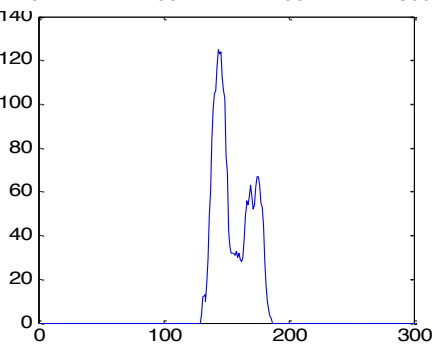

(b)

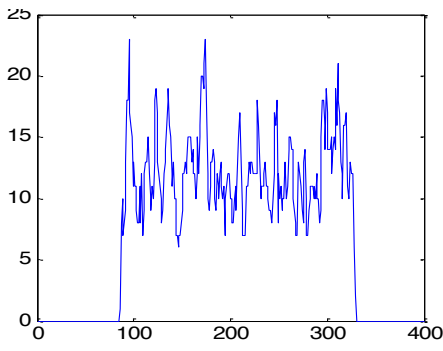

(c)

Fig. 3. Crack direction judgment. (a) Crack mark. (b) Line projection. (c) Column projection

Fig. 3 shows that the crack fig.3(a) are marked by the row and column projections in (b) and (c), respectively. These figures indicate the non-zero interval of the horizontal projection of the first vertical crack is $[0,300]$, and the nonzero interval of the vertical projection is [223, 285]. The length of the non-zero interval in the line projection of the vertical crack is longer than the column projection interval. Table.1 shows the non-zero interval list of the crack projection in Fig.3. Table.2 presents the crack parameter information.

Table 1. Horizontal and vertical projection intervals

\begin{tabular}{c|c|c}
\hline No. & Horizontal & Vertical \\
\hline 1 & {$[0,300]$} & {$[223,285]$} \\
2 & {$[0,300]$} & {$[281,368]$} \\
3 & {$[127,204]$} & {$[0,400]$} \\
4 & {$[124,179]$} & {$[79,328]$} \\
\hline
\end{tabular}

Table 2. Crack parameters

\begin{tabular}{c|c|c|c|c|c}
\hline No. & Area & Length & Maximum width & Minimum width & Shape judgment \\
\hline 1 & 3054 & 300 & 20 & 3 & 6 \\
3 & 3287 & 300 & 18 & 1 & Vertical crack \\
4 & 4379 & 400 & 27 & 4 \\
hertical crack & horizontal \\
\hline
\end{tabular}

\section{Conclusion}

To improve the problems of low efficiency, poor precision, and poor resistance to environmental interference in manual detection and traditional digital image processing methods, a novel method based on the machine vision and machine learning technologies was developed to detect the cracks. A case study was analyzed to eliminate noise areas and extract fracture information. The following conclusions are presented. (1) Multiscale Retinex filter improved by trilateral filter can improve the quality of image more effectively. Spectral clustering method solves the problem of clustering method by calculating the metric distance of all data. Mapping the relationship between high-dimensional data on twodimensional graph effectively reduces the dimension, and the crack segmentation is accurate and the details are clear.

(2) The interference region and noise point can be removed on the bases of the shape and contour features to ensure the long and linear features of the crack.

(3) Five features are employed in the SVDD classifier to establish the discriminant model of the cracks and non-cracks: width-to-length ratio, area ratio, ratio of the inscribed circle radius to the circumscribed circle radius, eccentricity of the connected domain, and filling degree of the connected domain The crack type can be judged by the projection of the crack on the horizontal and vertical coordinates.

This study combines image simulation experiment with theoretical research. The established crack detection model is simple and robust. The proposed model has certain reference value for the early detection of cracks and maintenance to avoid further damage. However, given that the image collection in this study is limited, covering all the noise conditions is impossible and the sample size is insufficient. In future studies, additional images should be collected continuously to improve the applicability and recognition accuracy of the proposed classifier.

\section{Acknowledgments}

This work was supported by the Xuzhou Science and Technology Plan Project(Grant No.KC18011 and KC19197), the Key Laboratory of Intelligent Industrial Control Technology of Jiangsu Province Research Project (Grant No.JSKLIIC201705), the Major Project of Natural Science Research of the Jiangsu Higher Education Institutions of China(Grant No.18KJA520012), the Natural Science Foundation of Jiangsu Province of China (Grant No.BK20161165), the Applied Fundamental Research Foundation of Xuzhou of China (Grant No.KC17072).

This is an Open Access article distributed under the terms of the Creative Commons Attribution License

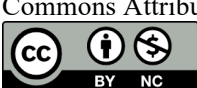




\section{References}

1. Ai, D., Jiang, G. Y., Siew, K. L., Li, C. W., "Automatic pixel-level pavement crack detection using information of multi-scale neighborhoods". IEEE Access, 6, 2018, pp. 24452 - 24463.

2. Teverovsky, A., "Insulation resistance and leakage currents in lowvoltage ceramic capacitors with cracks". IEEE Transactions on Components Packaging \& Manufacturing Technology, 4(7), 2014, pp. $1169-1176$.

3. Fan, Z., Wu, Y. M., Lu, J. W., et al., “Automatic pavement crack detection based on structured prediction with the convolutional neural network". Computer-Aided Civil and Infrastructure Engineering, 561(254), 2018, pp. 128-150.

4. Yang, X. C., Li, H., Yu, Y. T., et al., "Automatic pixel-level crack detection and measurement using fully convolutional network". Computer-Aided Civil and Infrastructure Engineering, 33(12), 2018 , pp. 1090-1109.

5. Badrinarayanan, V., Kendall, A., Cipolla, R., "Segnet: A deep convolutional encoder-decoder architecture for image segmentation". IEEE Transactions on Pattern Analysis and Machine Intelligence, 39(12), 2017, pp. 2481-2495.

6. Li, Q. Q., Liu, X. L., "Novel approach to pavement image segmentation based on neighboring difference histogram method". In: IEEE 2008 Congress on Image and Signal Processing, Sanya, China: IEEE, 2008, pp. 792-796.

7. Schmugge, S. J., Rice, L., Lindberg, J., et al., "Crack segmentation by leveraging multiple frames of varying illumination". In: 2017 IEEE Winter Conference on Applications of Computer Vision (WACV), California, USA: IEEE, 2017, pp. 1045-1053.

8. Achanta, R., Estrada, F., Wils, P., "Salient region detection and segmentation". In: Proceedings of the 6th International Conference on Computer Vision Systems, Santorini, Greece: IEEE, 2008, pp. 66 75 .

9. Achanta, R., Hemami, S., Estrada, F., Susstrunk S., "Frequencytuned salient region detection", In: IEEE Conference on Computer Vision and Pattern Recognition, Miami, USA: IEEE, 2009, pp.15971604.

10. Oliveira, H., Correia, P. L., "Automatic road crack detection and characterization". IEEE Transactions on Intelligent Transportation Systems, 14(1), 2013, pp. 155-168.

11. Nguyen, H. N., Kam, T. Y., Cheng, P. Y., "An automatic approach for accurate edge detection of concrete crack utilizing $2 \mathrm{~d}$ geometric features of crack". Journal of Signal Processing System, 77(3), 2014, pp. 221-240.

12. Zhang, L., Yang, F., Zhang, Y. D., et al., "Road crack detection using deep convolutional neural network". In: 2016 IEEE International Conference on Image Processing (ICIP). Arizona, USA: IEEE, 2016, pp. 3708-3712.

13. Valença, J., Dias-Da-Costa, D., Júlio, E., et al., “Automatic crack monitoring using photogrammetry and image processing". Measurement, 46(1), 2013, pp. 433-441.

14. Koch, C., Georgieva, K., Kasireddy,V., et al., "A review on computer vision based defect detection and condition assessment of concrete and asphalt civil infrastructure". Advanced Engineering Informatics, 29(2), 2015, pp.196-210.
15. Hu, Y., Zhao, C., "A local binary pattern based methods for pavement crack detection”. Pattern Recognition Research, 1(20103), 2010, pp. 140-147.

16. McCormack, K., Newman, T. K., Higley, J. D., et al., "Weighted neighborhood pixels segmentation method for automated detection of cracks on pavement surface images". Journal of Computing in Civil Engineering, 30(2), 2016, pp. 1-11.

17. Li, L. F., Sun, R. Q., "Bridge crack detection algorithm based on image processing under complex background". Advances in Laser and Optoelectronics, 56(06), 2019, pp. 112-122.

18. Prasanna, P., Dana, K. J., Gucunski, N., et al., "Automated crack detection on concrete bridges". IEEE Transactions on Automation Science \& Engineering, 13(2), 2016, PP. 591-599.

19. Zhao, S. S., He, N., "Road surface crack detection based on CNN". Transducer and Microsystem Technologies, 36(11), 2017, pp. 135138.

20. Cha,Y. J., Choi, W., Büyüköztürk, O., "Deep learning - based crack damage detection using convolutional neural networks". Computer-aided Civil \& Infrastructure Engineering, 32(5), 2017, pp. 361-378.

21. Zou, Q., Li, Q. Q., Mao Q. Z., et al., "Target-points MST for pavement crack detection". Geomatics and Information Science of Wuhan University, 36(1), 2011, pp. 71-75.

22. Wagdy, M., Faye, I., Rohaya, D., "Degradation enhancement for the captured document image using Retinex theory". In: International Conference on Information Technology and Multimedia at UNITEN, Barcelona, Spain: IEEE, 2015, pp. 363-367.

23. Niharika, E., Adeeba, H., Krishna, A. S. R., Yugander, P., "K-means based noisy SAR image segmentation using median filtering and otsu method". In: International Conference on IoT and Applications (ICIOT), Nagapattinam, India: IEEE, 2017.

24. Zhang, X.R., Song, Q., Gao, Z. Y., et al., "Spectral-spatial feature learning using cluster-based group sparse coding for hyperspectral image classification". IEEE Journal of Selected Topics in Applied Earth Observations and Remote Sensing, 9(9), 2016, pp. 4142 - 4159

25. Hinojosa, C. Bacca, J., Arguello, H., "Coded aperture design for compressive spectral subspace clustering”. IEEE Journal of Selected Topics in Signal Processing, 12 (6), 2018, pp. 1589 - 1600

26. Opbroek, A., Ikram, M. A., Vernooij, M. W. et al., "Transfer learning improves supervised image segmentation across imaging protocols". IEEE Transactions on Medical Imaging, 34(5), 2015, pp. $1018-1030$.

27. Ghesu, F. C., Krubasik, E., Georgescu, B., et al., "Marginal space deep learning: efficient architecture for volumetric image parsing". IEEE Transactions on Medical Imaging, 35 (5), 2016, pp.1217 1228.

28. Gao, J. C., Zhu Y. 1., Jia, Y. F., "Pattern recognition of unknown partial discharge based on improved SVDD". IET Science, Measurement \& Technology, 12(7), 2018, pp. 907 - 916.

29. Du, W. L., Tian, Y., Qian, F., "Monitoring for nonlinear multiple modes process based on LL-SVDD-MRDA". IEEE Transactions on Automation Science and Engineering, 11(4), 2014, pp. 1133 - 1148. 